\title{
Tally Education in Covid-19 Era: Impact on Health, Cancer Care and Education Sectors
}

\author{
Sunishtha Singh Yadav', Jayant Soni ${ }^{1}$, Shalini Porwal', Vandana Chauhan', \\ Shazia Rashid ${ }^{1}$, Ashish K Jain ${ }^{3}$
}

${ }^{1}$ Center for Medical Biotechnology, Amity Institute of Biotechnology, Amity University, Uttar Pradesh, India. ${ }^{2}$ Amity Institute of Microbial Technology, Amity University, Uttar Pradesh, India. ${ }^{3}$ Senior Research Fellow, ICAR, Avikanagar, India.

\begin{abstract}
The present paper will highlight the impact of COVID-19 on different sectors like cancer care and cancer patient's management and on the education system. With this pandemic, almost all the sectors have been affected badly but the major impact is visible on health care and education system because both these sectors demands face-to-face interactions as far as traditional approach is concerned. However, the good side of this event is several artificial intelligence-based methods have been evolved to minimize the impact of this pandemic on health care, research and education sector. This led to the evolution and revival of modern methods like telemedicine, video conferencing etc so that the motive of social distancing shall be fulfilled to avoid community spread of this deleterious infection. Different web platforms are being used for this purpose to name a few Google Meet, Zoom, Microsoft teams, WebEx and so forth. The ultimate goals however remain the same in both these sectors which is to meet the demand and supply, to provide assistance and to resolve the queries virtually without carrying out physical interactions.
\end{abstract}

Keywords: COVID-19- Telemedicine- cancer care- immunity- oncological societies

Asian Pac J Cancer Care, 5 (Suppl 1), 95-99

Submission Date: 06/12/2020Ａcceptance Date: 08/18/2020

\section{Introduction}

The coronaviruses which are human and animal pathogens marked the disastrous end of 2019 and sad beginning of 2020. The spread of this virus was initiated from Wuhan, a city in the Hubei Province of China. From epidemic this very soon changed to pandemic. This led to epidemic throughout the country and then followed by increased number of infected people across the globe [1-2]. The World Health Organization does the nomenclature of the disease as COVID-19 which stands for Coronavirus Disease 2019, in the month February 2020. This virus causes severe acute respiratory syndrome. Because of such a widespread of the disease global lockdowns happened around the globe and these events weaken down the health care system. Theses global cut offs presented several medical challenges globally in the health care management and especially in cancer care and management of cancer patients. The scenario of communication and interaction is now changing worldwide. COVID-19 era is actually redefining the policies and guidelines for governments, economic sectors, higher education sector, healthcare sector and research. Since then several guidelines have been issued by health ministries \& oncological societies for proper management of cancer patients in this pandemic, COVID-19's petrifying and dangerous effect has shaken the world to its core [3-4-5]. Moreover, as a measure to lower the spread of the COVID-19 pandemic, most governments around the world temporarily closed educational institutions so that the community interaction chances shall be minimized. In India too, the government as a part of the nationwide lockdown has closed majority of the private hospitals, all educational institutions, as a consequence of which, medical practitioners, oncologists and majority hospitals restricted the visiting hours and visitors \& learners ranging from school going children to postgraduate students, are affected. It has great impact on the thinking of almost all individuals worldwide and for

Corresponding Author:

Dr. Sunishtha Singh Yadav

Department Center for Medical Biotechnology, Amity Institute of Biotechnology, Amity University, Uttar Pradesh, India.

Email: singhsunishtha@gmail.com 
sure this radical change will be visible for longer period in the society [1-2]. To count the biggest transformation then that will be the one introduced in the health care system, research activities \& higher education sector because of COVID-19. In this the science of artificial intelligence \& deep learning models will play a very significant and crucial role. These branches of science will help in creating and customizing new methodologies for learning and imparting education \& information online. However, the implementation of these learning methods will come up with some challenges and will also demand innovations at regular intervals of time to overcome the shortcomings or ills generated during the course of administration. The post COVID-19 era, will not just led to antiquation of conventional way, however, will also provide blending of modern methods for patient's welfare and proper management; and also, methods of learning having both online and classroom teaching, as per the demand of the subject and curriculum. Though this combination will present some challenges as well in front of the health care \& education sector like how to match the demand of internet infrastructure so that hazzle free interactions and online classrooms shall be conducted [1-2-6-7].

\section{Challenges in Cancer patient's management in COVID-19 era}

Cancer patients have higher risk of developing severe consequences due to COVID-19. The interaction may lead the cancer patient's admission to ICU (intensive care unit) which may require invasive ventilation or death of the cancer patient may occur as compared with other disease patients. It did not establish a definitive increase in incidence of COVID-19 infection. The different challenges faced by the oncologist, cancer patients and managements units during this pandemic includes inadequate infrastructure; lack of management and skilled management unit and cancer care professionals [2-3-4-5]. This situation also disclosed the serious glitches in the supply-chain in cancer care across the world which resulted in compromised state regarding the safety and care of cancer patients. The cancer patient's management proved to be little more complexed during this pandemic because of lower immunity of the patients these are at higher risk of getting the infection. Another major impact of COVID-19 is on the translational research in the field of cancer which will lead to delay in the implementation of research data in cancer care advancement and critical care. The main objectives of the recommendations given by different health ministries and oncological societies is to control the adverse effects of COVID-19 pandemic on diagnosis, treatment and management of different forms of cancer across the world. The guidelines issued also suggested that some serious and realistic actions are to be taken to overcoming the challenges of treating cancer patients and to ensure their well-being in such a pandemic situation. Furthermore, these recommendations will guide the patients to decrease hospital time and interaction with medical practitioners. This era has revived the base of telemedicine in cancer care to provide video or telephonic consultation to cancer patients to be précised the telecommunication will support pre-treatment and follow-up meetings and appointments. The main motive of such regulations is to ensure telemedicine approach to decrease personal interactions by allowing extended electronic prescriptions so that repeated home-based oral cancer treatment shall be followed without the need for additional appointments during the pandemic [6-7-8-9-10].

\section{Rise of Digital Technology in cancer care and education} sectors

The most crucial challenge in the beginning of this pandemic was to deliver required care to cancer patients due to risks of severe outcomes and even death from combination of cancer and serious complications from COVID-19 as these groups of individuals are already immunocompromised. Because of which uncountable number of cancer patients suffered across the globe as the hospitals were either delaying or cancelling their surgeries and other procedures, like chemotherapy and radiation therapy. All this happened because of insufficient supplies of personal protective equipment (PPE) for health care providers, hospital capacities like ICUs were limited. Furthermore, seroprevalence data and lack of point-of-care testing also complicated the process [2-4-11-12]. Similarly, education sector faced number of problems since it too demands face to face interactions. Therefore, in health care institute, hospitals, educational institutes; data processing approaches, and online education technologies were required to operate in tandem due to the rapid adoption of digital technology caused by this lockdown. A significant number of these institutes are thinking about this as a perfect chance to analyse and send innovation to ensure that the training conveyance is conceivable and important. The pandemic has had a heavy impact on the shift from a one-to-one talk-based model to a digital one. This gap in the expression of instructions is putting pressure on the policymakers to find ways on how to have an e-learning arrangement that is commitment driven and would guarantee comprehensive learning. Any kind of change that is disruptive is also probable to deliver with it a few new opportunities that facilitate to convert the health care and higher education system globally and mainly in India which is making plans to convey the health care \& education system into a whole new level. To elaborate, post Covid-19 represents a chance to transform these sectors. Therefore, these sectors should take advantage of this opportunity to transform themselves. Introduction and revival of telemedicine, video conferencing in health care, curriculum design, collaborations, development of skills, and involvement of the staff - all should focus on internationalizing and globalization for better and effective outputs [1-2-7-10].

\section{Some of the key areas of opportunity are as follows}

\section{The rise in Blended Learning}

Health care and education sectors will move to a new model of realizing where both up close and 
personal conveyance alongside online models will turn into a standard. Additionally, this will require Medical practitioner/Lecturers to turn out to be innovatively further developed and experience some preparation to carry themselves to another level. In the area of telemedicine, patient's follow-up, curriculum development and pedagogy, new ways of assessing outcomes will have to be adopted which opens immense opportunities for a major transformation.

\section{Learning management}

For those companies that have been developing and strengthening learning management systems to be used so that great opportunity will be opened.

\section{Improvement in Learning Material}

This is an extraordinary open door for sectors to begin improving the nature of the learning material which is utilized in instructing and learning processes.

Since mixed learning will be the new arrangement of realizing there will be a push to discover better approaches to plan and convey content because of the way that utilization of learning the management system will get more openness and straightforwardness in health care and academics.

The National cancer grid took the rigorous initiative to share best practices and guidelines for cancer treatment during COVID-19 pandemic by organizing learning and informative webinars. They also established the open electronic-communication channels and a process for shared decision making, despite circumstances that preclude face-to-face meetings. This surely will be led to robust health technology assessment program, an essential tool in a country where public health care expenditures are low [1-2-7-10].

\section{Impact on Education system in India}

The UNESCO report estimates that more than 290 million students across 22 countries will suffer from coronavirus pandemic. UNESCO estimates that about 32 crores students are affected in India, including those in schools and colleges. Although the crisis is devastating, it makes our education system technologically advanced, but despite this, students and faculty have to make bigger adjustments because the learning method has always been physical. Many of them are not well equipped with technology tools to avail of remote learning. The traditional education system in India follows one-to-one based physical teaching, while a decade ago the advent of audio-visual aids was introduced in classrooms. Renowned Indian universities such as the University of Delhi also offer their students, online classes. But most of India's higher education institutions do not possess these high-end configurations. The presence of such an inconsistency, students are forced to be in a situation to face the impact which in turn would affect their performance for the whole academic year. Some bad Impacts of Corona Virus on Education System are:

\section{Cross-border Movement of Students}

Universities in many countries such as Australia, UK, Canada, and New Zealand are surprisingly dependent on the motion of students from China and India. So, its miles very clear that the cross-border movement will take the beating for the following 2-3 years and in the end can be leading to a fundamental economic risk for universities.

\section{Passive Learning}

As we realize that there is this unexpected move to web-based learning without appropriate arranging, which intensely influences nations like India where the spine for web-based learning was not prepared, and educational program was not intended for such an arrangement. Likewise, web-based learning could be a dull arrangement of instructing as it is making another arrangement of uninvolved students which can present new difficulties.

\section{Unprepared Teachers/Lecturers}

Online teaching is a very unique type of technique and now, not all instructors are suitable at it or not all of them are equipped for this type of unexpected change from face to face learning to an online one. Potential outcomes are there that in such circumstances, learning results may not be accomplished and it might be just bringing a distraction to the students [1-9].

Gearing up of diversified and interdisciplinary collaboration in post COVID-19 era

This whole situation has illustrated that we are globally interconnected and when it is so then it is significant to understand the inter-relatedness in diversified and interdisciplinary work across boundaries and work in a globally collaborative way. The interdisciplinary collaborations will be beneficial for the professionals, organizations and to the public, private \& government systems. These collaborative initiatives will strengthen education and research systems, stakeholder's satisfaction, acceptance of new implementations and improved outcomes. This will gear up diversified and interdisciplinary collaboration in COVID-19 and post COVID-19 era will also integrate "the input-processesoutput approach". This concept will help to deepen theoretical understanding and will also resolve the challenges in analysing the current state of knowledge. It provided the rationale for this systematic review. The invasion of COVID-19 has a tremendous impact on different strata of the society in several ways and has shown the importance and benefits of multi-sectorial collaborations. And will prove the benefits of sharing of expertise across different groups world-wide to come up with enhanced and effective outputs. The policy-makers will now focus on interprofessional collaborations which will be based on the infrastructure of the educational landscape. The main focus of these collaborations will be centralization of information gathered through research, maintaining the time lines for effectiveness, new safety regulations to be published (mandatory use of personal protective equipment; PPE) and maintaining enhanced 
efficiency and equity. These collaborations will enhance synergy between digital and in-person training modalities to ensure that critical outcome of research and education shall be communicated across world. These collaborations will allow integration of diversified and interdisciplinary approach into the routine of research and education and will ensure highest-yield activity. Both resilience and adaptability will induce and enhance interdisciplinary collaborations in post COVID-19 era. This era will give more importance to "one health concept", which is define by WHO as an approach to designing and implementing programmes, policies, legislation and research in which multiple sectors communicate and work together to achieve better public health outcomes [10-11-12-13-14].

New methods of diagnosis \& prognosis in cancer care and assessment and evaluation in education sector (service and technology combination)

This era has given new horizons to the combination of service and technology. Several innovative methods have been explored for assessment and evaluation related to different domains including cancer care and education sector. Though technology has always influenced these two sectors majorly, however COVID-19 situation has redefined the different intersects where technology shall be implemented during process like diagnosis and prognosis in cancer care; and assessment and evaluation in education sector. This new era has marked the beginning of exploration of untouched spheres of technology. It is much about technology reaching to people (cancer patients and students) in the most effective and friendly ways with better outcomes [1-2-3-4-5]. These implications are procedures/processes accessible from distant locations, equally appropriate by incorporating vivid simulations of real-world situations. The new ways invented during this era are more flexible, responsive and contextual; and will be followed for next several decades. This has evolved next generation of service and technology combination through better incorporation and management of timing, accessibility, pathways, feedbacks and item types as compared to traditional methods. Incorporation of more and more computing and telecommunications devices has a major lay down in this era. This will be much about digital literacy and its implementation. These new methods will be combination of both synchronous and asynchronous. The main purpose of these implementations is to minimize personal or face-to-face contact, and increase virtual interactions through telephonic assistance or video consultations, particularly for pre-treatment meetings and follow-up appointments in cancer care. Both these methods have their own advantages \& disadvantages as well. But the motive of both these methods on education sector is to impart required and necessary information through incorporation of different digital platforms [6-7-8-9]. Online instruction is led in two different ways. The first is using recorded information, which, when opened out to open, are alluded to as Massive Open Online Course (MOOCs). The subsequent one is utilizing live online directed as online classes, or zoom meetings [10-11-12-13-14].
In conclusion, COVID-19 is a global threat which is affecting the lives worldwide. It is caused by novel Corona virus called SARS-COV-2. At present there is no possible treatment for covid-19. However, the chances of infection can be minimized by boosting immunity against virus. This pandemic has not only affected the lifestyles but also the economy of the countries across the globe, the health care sector and the education sector. It has cause global socio-economic depression. But out of all the negative effect of coronavirus it has some positive sides as it contributed majorly to the digitalization or artificial intelligence. Health care sector is now switching to telemedicine concept in cases where of chronical diseases treatment like cancer where it is known that the patient's immunity is already compromised and therefore any kind of public exposure can be hazardous. Similarly, education sector has evolved a lot by adopting to artificial intelligence: synchronous and asynchronous methods of teaching. Globalization in field of health care and education sectors is the demand of this era. Collaborative works in field of research, health care and education will be more acceptable in this era.

\section{References}

1. Arora AK, Srinivasan R. Impact of Pandemic COVID-19 on the Teaching - Learning Process: A Study of Higher Education Teachers. Prabandhan: Indian Journal of Management. 202004 30;13(4):43. https://doi.org/10.17010/pijom/2020/ v13i4/151825

2. Boettler T, Newsome PN, Mondelli MU, Maticic M, Cordero E, Cornberg M, Berg T. Care of patients with liver disease during the COVID-19 pandemic: EASL-ESCMID position paper. JHEP Reports. 2020 06;2(3):100113. https://doi. org/10.1016/j.jhepr.2020.100113

3. Cannistra SA, Haffty BG, Ballman K. Challenges Faced by Medical Journals During the COVID-19 Pandemic. Journal of Clinical Oncology. 202007 01;38(19):2206-2207. https:// doi.org/10.1200/jco.20.00858

4. Huang C, Wang Y, Li X, Ren L, Zhao J, Hu Y, Zhang L, Fan G, Xu J, Gu X, Cheng Z, Yu T, Xia J, Wei Y, Wu W, Xie X, Yin W, Li H, Liu M, Xiao Y, Gao H, Guo L, Xie J, Wang G, Jiang R, Gao Z, Jin Q, Wang J, Cao B. Clinical features of patients infected with 2019 novel coronavirus in Wuhan, China. The Lancet. 2020 02;395(10223):497-506. https:// doi.org/10.1016/s0140-6736(20)30183-5

5. Liang W, Guan W, Chen R, Wang W, Li J, Xu K, Li C, Ai Q, Lu W, Liang H, Li S, He J. Cancer patients in SARSCoV-2 infection: a nationwide analysis in China. The Lancet Oncology. 2020 03;21(3):335-337. https://doi.org/10.1016/ s1470-2045(20)30096-6

6. Lone A. Impact of online education in India. IJESC. 2017;7(7):13050-952.

7. Pramesh C, Badwe RA. Cancer Management in India during Covid-19. New England Journal of Medicine. 202005 14;382(20):e61. https://doi.org/10.1056/nejmc2011595

8. Schreiber RD, Old LJ, Smyth MJ. Cancer Immunoediting: Integrating Immunity's Roles in Cancer Suppression and Promotion. Science. 201103 24;331(6024):1565-1570. https://doi.org/10.1126/science.1203486

9. UNESCO-COVID-19. Education response. Education sector issue note. April 2020. https://unesdoc.unesco.org/ ark:/48223/pf0000373305..

10. WHO main website. www.who.int. Date last accessed: 
March 10, 2020.

11. World Health Organization. Director-General's remarks at the media briefing on $2019-\mathrm{nCoV}$ on 11 February 2020. https://www.who.int/dg/speeches/detail/who-directorgeneral-s-remarks-at-the-media-briefing-on-2019-ncov-on11-february-2020 (Accessed on February 12, 2020).

12. World Health Organization. Novel Coronavirus (2019-nCoV) situation reports. www.who.int/emergencies/diseases/novelcoronavirus-2019/situation-reports/. Date last accessed: March 10, 2020.

13. Xu Z, Shi L, Wang Y, Zhang J, Huang L, Zhang C, Liu S, Zhao P, Liu H, Zhu L, Tai Y, Bai C, Gao T, Song J, Xia P, Dong J, Zhao J, Wang F. Pathological findings of COVID-19 associated with acute respiratory distress syndrome. The Lancet Respiratory Medicine. 2020 04;8(4):420-422. https:// doi.org/10.1016/s2213-2600(20)30076-x

14. Yu J, Ouyang W, Chua MLK, Xie C. SARS-CoV-2 Transmission in Patients With Cancer at a Tertiary Care Hospital in Wuhan, China. JAMA Oncology. 202007 01;6(7):1108. https://doi.org/10.1001/jamaoncol.2020.0980

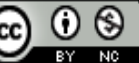

This work is licensed under a Creative Commons AttributionNon Commercial 4.0 International License. 